\title{
Ulrich Ahrens
}

\section{Margitta Beil-Hildebrand: Institutional Excellence im Krankenhaus- Rhetorik und Realität}

\author{
Hans Huber, Bern (ISBN 3-456-83974-X), 29,95 EUR
}

Online veröffentlicht: 27 März 2004

(C) Springer-Verlag 2004

Kaum ein anderer Bereich sieht sich momentan einem ähnlich starken Veränderungsdruck ausgesetzt wie der Krankenhaussektor in Deutschland. Die Einführung von Diagnosis Related Groups, das Urteil des Europäischen Gerichtshofs zum Bereitschaftsdienst, budgetäre Nullrunden, hohe Investitionslücken durch die dualistische Finanzierung und andere Spannungsfelder bedingen einen unumkehrbaren Wandel im Krankenhaus. Aufgrund der veränderten Rahmenbedingungen befassen sich viele Arbeiten zu diesem Thema mit der zunehmenden Ökonomisierung des stationären Sektors und den daraus abzuleitenden strategischen und operativ-organisatorischen Handlungsoptionen für die Kliniken. Wie sich diese neuen Zielsetzungen konkret auswirken, wie der potenziell stärker zutage tretende Konflikt zwischen Verwaltung auf der einen Seite und Ärzten und Pflegekräften auf der anderen Seite zu bewältigen ist, wird weitaus weniger intensiv diskutiert. An diesem Punkt setzt die ethnographische Feldstudie der Autorin Beil-Hildebrand an, in der sie die Unternehmenskultur eines Allgemeinkrankenhauses einer kritischen Analyse unterzieht. Dabei untersucht sie, inwieweit die von der Krankenhausleitung vermittelten Werte und Mitgestaltungsmöglichkeiten von den Mitarbeiter(inne)n angenommen und umgesetzt werden, um die formulierten medizinischen und wirtschaftlichen Organisationsziele zu erreichen. Nach Angabe der Autorin zielt die Studie gemäß den ethnographischen Grundsätzen nicht auf die Beantwortung einer wissenschaftlichen Frage ab, sondern will die Schaffung und Steuerung einer Unternehmenskultur als rhetorischen und praktischen Mechanismus auffassen. Hierdurch werden auch die Hauptadressaten dieser Studie determiniert: Akteure bzw. Praktiker im Krankenhaus.

Das Buch ist in drei Hauptabschnitte unterteilt. Zuerst bietet es einen umfassenden theoretischen Überblick zur
Organisationskultur und einen ethnographischen Ansatz zur Arbeitsprozessanalyse innerhalb von zwei Kapiteln, bevor die Schilderung der Rahmenbedingungen und der Vorgehensweise der Studie Inhalt der folgenden zwei Kapitel ist. Die restlichen sechs Kapitel widmen sich der eigentlichen Untersuchung im Krankenhaus und schließen mit einer Diskussion und Zusammenfassung der wichtigsten Ergebnisse ab.

Die behandelten Themengebiete bieten einen riesigen Fundus an Literaturverweisen nationaler und internationaler Färbung und werden mit zahlreichen-teils sehr langen-Zitaten vorgetragen. Die Fülle des Materials, die für den fachfremden Leser mancherorts erdrückend erscheinen mag, wird durch die knappen Zusammenfassungen am Ende jedes Kapitels nochmals in bemerkenswerter Form aufgearbeitet. Allerdings hätten einige Abbildungen zu einer Auflockerung des Textes und zum allgemeinen Verständnis beigetragen. Die stringente Struktur und Herleitung der Schlussfolgerung, dass eine „Diskrepanz zwischen Rhetorik und Realität“ im Krankenhaus mit ihren negativen Auswirkungen auf Mitarbeiterverhalten und -motivation besteht, greift schlussendlich aber etwas zu kurz. Die Empfehlungen zur Verbesserung der hier betrachteten Umstände wie ,die Entwicklung von Führungskräften, die in der Lage sind, Reorganisationen und Innovationen $\mathrm{zu}$ managen" sind eher generischer Natur und hätten eine ausführlichere Betrachtung verdient.

Trotzdem gelingt es der Autorin mit diesem Ansatz eindrucksvoll, die Bedeutung nichtpekuniärer Faktoren für den Erfolg einer Organisation-hier am Beispiel des Krankenhauses-zu unterstreichen. Dieser Denkanstoß mag vielen beteiligten Akteuren helfen, dem Wandel vielschichtiger und erfolgversprechender zu begegnen.

U. Ahrens (-

Bain Company Germany Inc.,

Karlsplatz 1, 80335 München

E-Mail: ulrich.ahrens@bain.com 